\title{
Mutation of ATF6 causes autosomal recessive achromatopsia
}

\author{
Muhammad Ansar ${ }^{1,2} \cdot$ Regie Lyn P. Santos-Cortez ${ }^{1} \cdot$ Muhammad Arif Nadeem Saqib $^{2}$. \\ Fareeha Zulfiqar $^{3} \cdot$ Kwanghyuk Lee $^{1} \cdot$ Naeem Mahmood Ashraf $^{2} \cdot$ Ehsan Ullah $^{2} \cdot$ Xin Wang $^{1}$. \\ Sundus Sajid ${ }^{2} \cdot$ Falak Sher Khan $^{2} \cdot$ Muhammad Amin-ud-Din $^{4} \cdot$ University of Washington Center for \\ Mendelian Genomics · Joshua D. Smith ${ }^{5} \cdot$ Jay Shendure $^{5} \cdot$ Michael J. Bamshad $^{5} \cdot$ Deborah A. Nickerson $^{5}$. \\ Abdul Hameed $^{6} \cdot$ Saima Riazuddin $^{3} \cdot$ Zubair M. Ahmed $^{3} \cdot$ Wasim Ahmad $^{2} \cdot$ Suzanne M. Leal $^{1}$
}

Received: 3 April 2015 / Accepted: 19 May 2015 / Published online: 11 June 2015

(C) The Author(s) 2015. This article is published with open access at Springerlink.com

\begin{abstract}
Achromatopsia (ACHM) is an early-onset retinal dystrophy characterized by photophobia, nystagmus, color blindness and severely reduced visual acuity. Currently mutations in five genes $C N G A 3, C N G B 3, G N A T 2$, $P D E 6 C$ and $P D E 6 H$ have been implicated in ACHM. We performed homozygosity mapping and linkage analysis in a consanguineous Pakistani ACHM family and mapped the locus to a $15.12-\mathrm{Mb}$ region on chromosome 1q23.1-q24.3 with a maximum LOD score of 3.6. A DNA sample from an affected family member underwent exome sequencing. Within the ATF6 gene, a single-base insertion variant c.355_356dupG (p.Glu119Glyfs*8) was identified, which
\end{abstract}

The members of the University of Washington Center for Mendelian Genomics are listed in "Appendix" section.

Electronic supplementary material The online version of this article (doi:10.1007/s00439-015-1571-4) contains supplementary material, which is available to authorized users.

Suzanne M. Leal

sleal@bcm.edu

1 Department of Molecular and Human Genetics, Center for Statistical Genetics, Baylor College of Medicine, 1 Baylor Plaza 700D, Houston, TX 77030, USA

2 Department of Biochemistry, Faculty of Biological Sciences, Quaid-i-Azam University, Islamabad 45320, Pakistan

3 Department of Otorhinolaryngology Head and Neck Surgery, School of Medicine, University of Maryland, Baltimore, MD 21203, USA

4 University of Education, Dera Ghazi Khan Campus, Lahore 32200, Pakistan

5 Department of Genome Sciences, University of Washington, Seattle, WA 98195, USA

6 Institute of Biomedical and Genetic Engineering, Islamabad 44000, Pakistan completely segregates with the ACHM phenotype within the family. The frameshift variant was absent in public variant databases, in 130 exomes from unrelated Pakistani individuals, and in 235 ethnically matched controls. The variant is predicted to result in a truncated protein that lacks the DNA binding and transmembrane domains and therefore affects the function of ATF6 as a transcription factor that initiates the unfolded protein response during endoplasmic reticulum (ER) stress. Immunolabeling with anti-ATF6 antibodies showed localization throughout the mouse neuronal retina, including retinal pigment epithelium, photoreceptor cells, inner nuclear layer, inner and outer plexiform layers, with a more prominent signal in retinal ganglion cells. In contrast to cytoplasmic expression of wild-type protein, in heterologous cells ATF6 protein with the p.Glu119Glyfs $* 8$ variant is mainly confined to the nucleus. Our results imply that response to ER stress as mediated by the ATF6 pathway is essential for color vision in humans.

\section{Introduction}

Achromatopsia (ACHM) is an autosomal recessive heterogeneous disorder characterized by symptoms that include the inability to discriminate colors, reduced visual acuity primarily in daylight, nystagmus and severe photophobia (Michaelides et al. 2004). Initially, ACHM was considered as a stationary disorder but recent imaging studies have shown the progressive nature of disease in several patients (Thiadens et al. 2010; Aboshiha et al. 2014). Its prevalence is estimated to be about 1 in 30,000 individuals worldwide. To date, mutations in CNGA3 [MIM 600053], CNGB3 [MIM 605080], GNAT2 [MIM 139340], PDE6C [MIM 600827] and PDE6H [MIM 601190] are known to cause ACHM (Kohl et al. 1998, 2000, 2002, 2012; Chang 
et al. 2009). The proteins encoded by these genes are conespecific and have critical roles in the phototransduction cascade occurring in the cone photoreceptors. CNGA3 and $C N G B 3$ mutations are the major cause of ACHM worldwide, with mutations in GNAT2, PDE6C and PDE6H playing a lesser role (Kohl et al. 2012). However, the known gene mutations do not account for all cases of ACHM (Saqib et al. 2011).

Using homozygosity mapping and linkage analysis, we mapped a new locus for ACHM in a consanguineous Pakistani family. Within the mapped region, from exome sequence data we identified a frameshift variant c.355_356dupG (p.Glu119Glyfs*8) in the ATF6 [MIM 605537] gene which encodes cyclic AMP-dependent activating transcription factor- 6 alpha. The p.Glu119Glyfs*8 variant affects the targeting of ATF6 protein in heterologous cells. Immunostaining revealed ubiquitous expression of ATF6 in the neuronal retina. Our results highlight the importance of the ATF6-mediated signaling pathway to color vision in humans.

\section{Materials and methods}

\section{Subjects}

This study was initiated after obtaining approval from the institutional review boards of Quaid-i-Azam University and Baylor College of Medicine and Affiliated Hospitals. Informed consent was obtained from all participating individuals. Blood samples were collected from four affected and seven healthy individuals (Fig. 1a) and genomic DNA was extracted with GenElute ${ }^{\mathrm{TM}}$ blood genomic DNA kit (Sigma-Aldrich, St. Louis, MO, USA). A detailed interview was conducted with family members to gather information on pedigree structure, comorbidities, onset of disease and initial symptoms. The clinical diagnosis was based on presenting symptoms, physical examinations, visual acuity measurement, fundoscopy, color vision test and full-field electroretinography (ffERG).

\section{Genotyping and linkage analysis}

DNA samples from 11 family members underwent a genome-wide scan using the Infinium ${ }^{\circledR}$ HumanCoreExome BeadChip (Illumina, USA) which contains $\sim 550 \mathrm{~K}$ single-nucleotide polymorphism (SNP) markers. The resulting genotype data were analyzed using PedCheck (O'Connell and Weeks 1998) to identify Mendelian inconsistences and MERLIN (Abecasis et al. 2002) to detect double recombination events, which occur within small physical distances that are most likely due to genotyping errors. Markers with suspected errors were removed from subsequent analyses. HomozygosityMapper (Seelow et al. 2009) was used to identify regions of homozygosity. Two-point and multipoint parametric linkage analyses were performed using Superlink (Silberstein et al. 2006) and MERLIN respectively, using a fully penetrant autosomal recessive mode of inheritance with no phenocopies and a disease allele frequency of 0.001. Allele frequencies for SNP markers were estimated from the founders and reconstructed founders from 16 Pakistani families for whom genotype data were obtained using the same array. For multipoint linkage analysis, genetic map positions were obtained through interpolation from physical maps to the Rutgers combined linkage-physical map build 37 (Matise et al. 2007).

\section{Exome sequencing}

Exome sequencing was performed using a DNA sample from affected individual III-1 (Fig. 1a). Sequence capture was performed with the Roche NimbleGen SeqCap EZ Human Exome Library v.2.0 to target $36.6 \mathrm{Mb}$ of sequence. Sequencing was carried out on an Illumina HiSeq and fastq files were aligned to the human reference sequence (hg19) using Burrows-Wheeler Aligner ( $\mathrm{Li}$ and Durbin 2009). Realignment of regions containing indels, recalibration of base qualities, and variant detection and calling were carried out using the Genome Analysis Toolkit (McKenna et al. 2010). Annotation of variant sites was performed using SeattleSeq 137.

The exome data were analyzed to find within the region of homozygosity coding or splice variants which are not in $\mathrm{dbSNP}$ and had a minor allele frequency (MAF) $<0.05 \%$ in the Exome Aggregation Consortium (ExAC) database which includes data from $>60,000$ next-generation sequences. Occurrence of the ATF6 c.355_356dupG variant was also examined using exomes from 130 unrelated Pakistani individuals who are affected with a Mendelian trait but do not have eye-related phenotypes.

\section{Mutation analysis}

Sanger sequencing was used to confirm the co-segregation of the c.355_356dupG variant in exon 5 of ATF6 (NM_007348.3) with ACHM within the family using primers $5^{\prime}$ GGAAGAAATGTAGCAACAGATCAA $3^{\prime}$ and 5'CCAGTGACAGGCTTATCTTCC $3^{\prime}$. DNA samples from 235 Pakistani control individuals were also screened for the ATF6 variant. Amplified PCR products were purified by ExoSAP-IT (Affymetrix, Cleveland, OH, USA) and sequenced using BigDye Terminator v3.1 Cycle Sequencing Kit (Life Technologies, Carlsbad, CA, USA). Sequence data were analyzed with Sequencher 4.9 (Gene Codes Corporation, Ann Arbor, MI, USA). 
Fig. 1 Pedigree drawing, clinical findings, chromatograms and RT-PCR expression results for ATF6. a Pedigree drawing of MA28 family with autosomal recessive ACHM. Genotypes for the ATF6 c.355_356dupG variant are shown below each symbol of corresponding family members with available DNA samples. b Fundoscopy images and ERG data for affected proband III-1 (arrow in panel a) which shows bilateral loss of the foveal reflex and reduced cone response, respectively. Other retinal features are normal, including a palecentered optic disc with clear borders, 0.3-0.4 cup-to-disc ratio, and no arteriovenous nicking, hemorrhages, emboli or infarcts. c Chromatograms of family members who are homozygous, heterozygous and wild-type for the ATF6 variant. d RT-PCR results showed the presence of multiple ATF6 isoforms in human eye and retinal pigment epithelium cells. I NM_007348.3 exons 1-16, 2028 bp; II NM_007348.3 exons 15-16, $265 \mathrm{bp}$; III AB208929 exon 14, 190 bp. Table S1 has additional details on isoforms and exons tested. GAPDH (143 bp) was used as internal control. Lanes: 1 100bp leader; 2, 5, 8 whole eye; 3 , 6,9 retinal pigment epithelium; $4,7,10$ negative control

(a)

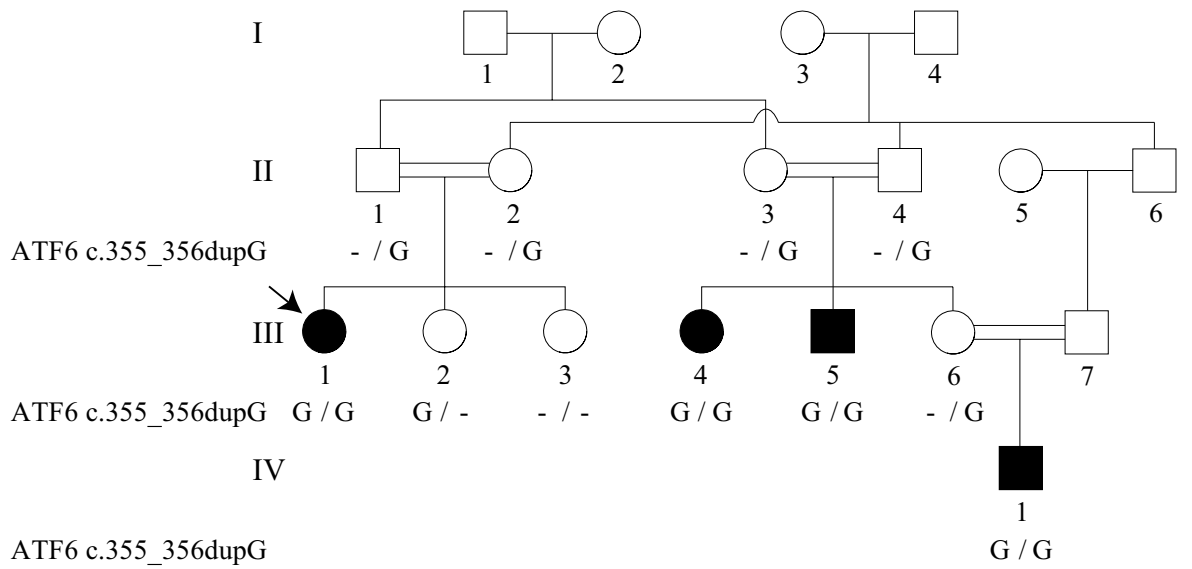

(b)

Fundus
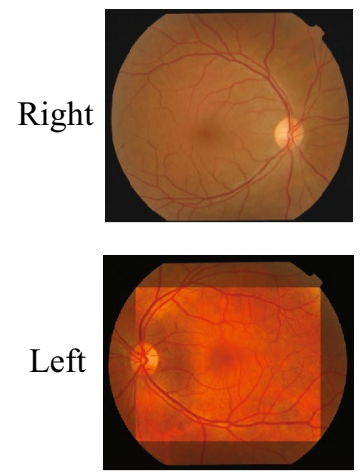

(c)

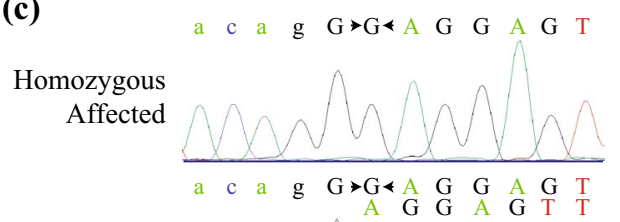

Heterozygous

Carrier

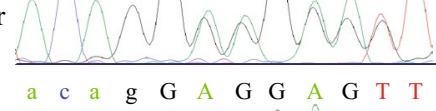

Homozygous

Unaffected
Scotopic Rod Response

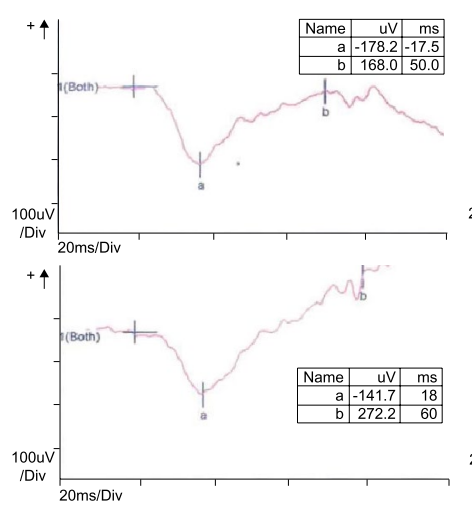

Photopic Cone Response

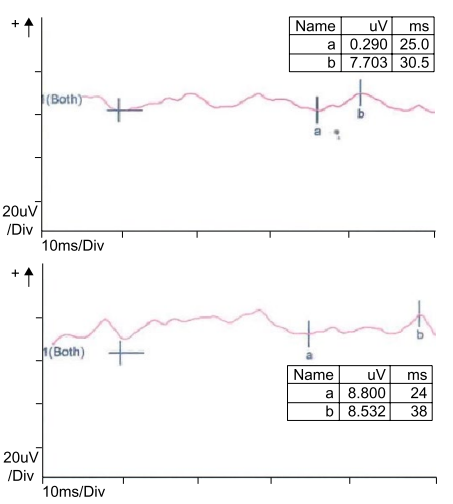

(d)

ATF6 cDNA Isoforms

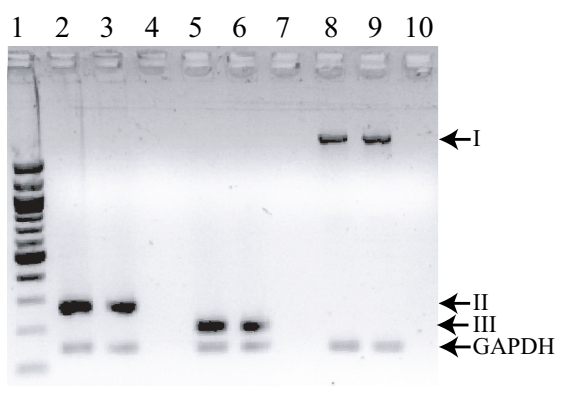

Multiple ATF6 and ATF6-like protein sequences were derived by blastp from the UniProt database and aligned using Clustal Omega (Sievers et al. 2011). Molecular modeling of wild-type and mutant ATF6 was performed using Phyre2 (Kelley and Sternberg 2009) with CREB protein structure as template (Protein Data Bank ID 1DH3).

\section{cDNA synthesis and reverse transcription (RT)-PCR}

To check the expression of ATF6 in the eye, total RNA samples were obtained for human eye (Biochain Institute, Newark, CA, USA) and retinal pigment epithelial cells (ScienCell
Research Laboratories, Carlsbad, CA, USA). cDNA was synthesized using $1 \mu \mathrm{g}$ RNA, SMARTScribe Reverse Transcriptase (Clontech, Mountain View, CA, USA) and random hexamers (Invitrogen, Carlslbad, CA, USA). Multiple primer sets (Table S1) were used to detect the expression of ATF6 isoforms in the RNA samples from human eye and retinal pigment epithelium cells. GAPDH was used as internal control.

\section{Western blot analysis}

All animals used in this study were kept with the approval of the Institutional Animal Care and Use Committee of the 
University of Maryland, School of Medicine. C57BL/6 mouse retinae were fixed in $4 \%$ paraformaldehyde for $30 \mathrm{~min}$, washed in PBS, and homogenized and sonicated in ice-cold RIPA lysis buffer (Teknova R3792, Hollister, CA, USA) supplemented with protease inhibitor cocktail (Sigma-Aldrich P8340, St. Louis, MO, USA). Proteins were extracted and denatured by boiling at $95{ }^{\circ} \mathrm{C}$ for $5 \mathrm{~min}$ in SDS-PAGE sample buffer $(0.125 \mathrm{M}$ Tris- $\mathrm{HCl}, 20 \%$ glycerol, $4 \%$ SDS, $0.005 \%$ bromophenol blue). A 50- $\mu \mathrm{g}$ protein sample was separated on a 4-20\% gradient Trisglycine gel (Novex, San Diego, CA, USA) and transferred to nitrocellulose membrane, blocked overnight at $4{ }^{\circ} \mathrm{C}$ with $5 \%$ dry milk in TBST (10 mM Tris-HCl pH 7.5, $150 \mathrm{mM}$ $\mathrm{NaCl}, 0.05 \%$ Tween 20), and stained with anti-ATF6 antibodies (1:500 in blocking solution) for $1 \mathrm{~h}$ at room temperature. After three washes, the membranes were incubated in a 1:1500 dilution of horseradish peroxidase-conjugated anti-rabbit secondary antibody (GE Healthcare) for $1 \mathrm{~h}$ at room temperature and developed using the ECL Plus Western Blotting Detection system (Amersham Pharmacia Biotech, Arlington Heights, IL, USA).

We also performed western blot analysis to study the steady state level of wild-type and mutant ATF6 proteins in transfected COS-7 cells. After $24 \mathrm{~h}$ of transfection, COS-7 cells were collected and mixed with $2 \times$ sample loading buffer with reducing agent and processed for western blot analysis as described above.

\section{Immunostaining, expression plasmid and localization assay}

Cryo-preserved retinal sections from adult CD1 mice were rehydrated in PBS with $0.1 \%$ Triton X-100 for $20 \mathrm{~min}$. After blocking in $10 \%$ Normal Goat Serum (NGS) for $45 \mathrm{~min}$ at room temperature, sections were incubated with various concentrations of anti-ATF6 antibodies (abcam, Cambridge, MA, USA) in a dark humid chamber for $3 \mathrm{~h}$, followed by washing in $1 \mathrm{X}$ PBS and conjugation with Alexa 488 secondary antibodies (Life Technologies, Carlsbad, CA, USA). For nuclear DNA, we used 4'6-diamidino2-phenylindole (DAPI; $2 \mu \mathrm{g} / \mathrm{ml}$ ). Actin was decorated with either rhodamine phalloidin (1:500) or Alexa 647-phalloidin (1:100). Sections were mounted with ProLong Gold antifade reagent (Molecular Probes, Life Technologies) and imaged using LSM510 (Zeiss, Pleasanton, CA, USA).

Human full-length (Accession no. NM_007348) ATF6 flag-tagged and eGFP-tagged cDNA constructs were purchased from GeneCopoeia (Rockville, MD). To insert the c.355_356dupG mutation, we performed site-directed mutagenesis on the construct using the QuikChange Lightening Kit (Agilent Technologies, Santa Clara, CA). To study expression and targeting, we transfected COS-7 cells, grown in DMEM that was supplemented with $10 \%$ FBS, with flag-tagged wild-type and mutant ATF6 constructs. Transfected cells were maintained at $37{ }^{\circ} \mathrm{C}$ in $5 \% \mathrm{CO}_{2}$ for $24 \mathrm{~h}$. After incubation, transfected cells were fixed with $4 \%$ paraformaldehyde (EMS) in PBS for $30 \mathrm{~min}$, permeabilized for $15 \mathrm{~min}$ in $0.2 \%$ Triton X-100 and nonspecific binding sites were blocked by incubation in $2 \%$ BSA and $5 \%$ normal goat serum in PBS for 30 min. COS-7 cells were incubated for $2 \mathrm{~h}$ at room temperature with anti-flag (1:500) and primary antibodies for Calregulin (1:500) as endoplasmic reticulum (ER) marker, followed by incubation with Alexa Fluor 488 goat anti-mouse and Alexa Fluor 546 goat anti-rabbit secondary antibodies (Molecular Probes) for $45 \mathrm{~min}$ at room temperature. Rhodamine phalloidin was used to label actin cytoskeleton, while nuclei were decorated with DAPI. Samples were washed in PBS, mounted using ProLong Gold Antifade reagent (Molecular Probes), and imaged with the use of a LSM510 coreduo confocal microscope equipped with a Zeiss $63 \mathrm{X}$ objective (Zeiss Microimaging).

\section{Antibody validation}

To validate anti-ATF6 antibody (ab37149; abcam, Cambridge, MA, USA), we performed a colocalization assay using flag-tagged ATF6-transfected COS-7 cells. The cells were grown on coverslips in Dulbecco's modified Eagle's medium (Invitrogen) supplemented with $10 \%$ fetal bovine serum and then transfected with Lipofectamine reagent (Invitrogen). After incubation at $37{ }^{\circ} \mathrm{C}$ with $5 \% \mathrm{CO}_{2}$ for $24 \mathrm{~h}$, transfected cells were fixed with $4 \%$ paraformaldehyde (EMS) in PBS for $30 \mathrm{~min}$, permeabilized for 15 min in $0.2 \%$ Triton X-100 and nonspecific binding sites were blocked by incubation in $2 \%$ BSA and $5 \%$ normal goat serum in PBS for 30 min. Primary ATF6 and anti-flag antibodies were diluted in blocking solution to a final concentration of $\sim 0.5 \mu \mathrm{g}$ per ml. COS-7 cells were incubated for $2 \mathrm{~h}$ at room temperature with ATF6 and anti-flag antibodies, followed by incubation with Alexa Fluor 546 goat anti-rabbit and Alexa Fluor 488 goat anti-mouse secondary antibodies (Molecular Probes) for $45 \mathrm{~min}$ at room temperature. Rhodamine phalloidin labeled the actin cytoskeleton and DAPI was used to stain nuclei. Samples were washed in PBS, mounted using ProLong Gold Antifade reagent (Molecular Probes), and imaged with the use of a LSM510 coreduo confocal microscope.

\section{Results}

The four affected individuals (III-1, III-4, III-5, IV-1; Fig. 1a) of family MA28 presented with severe photophobia, nystagmus and absence of color discrimination from early childhood. They have normal night vision, but in 
daylight they experience extreme discomfort and exhibit extensive blinking. Complete physical examination and standard blood tests (e.g., blood cell counts, lipid profile, liver function tests, glucose, creatinine, among others) of all four affected individuals ruled out the presence of additional comorbidities (i.e., neurologic, otolaryngologic, hematologic, cardiovascular, respiratory, gastrointestinal, endocrine, urinary) and substantiated the presence of isolated achromatopsia. The visual acuity measurements of the affected individuals range from 0.08 to 0.2 . The Ishihara 24-plate color test revealed severe defects in all affected individuals. Fundus examination of the proband III-1, at the age of 16 years, was normal except for loss of the foveal reflex (Fig. 1b). The ffERG of the same individual was typical of ACHM, i.e., (1) normal amplitude and latency of scotopic or rod response in dark-adapted phase at $\leq 30 \mathrm{~Hz}$ but subnormal rod response at $120 \mathrm{~Hz}$, (2) absent photopic flicker response with loss of regular sinusoidal waveforms, and (3) normal latency but severely diminished amplitude of photopic or single cone flash response (Fig. 1b, S1). Additionally, oscillatory potentials were demonstrated during scotopic responses at $30 \mathrm{~Hz}$ (Fig. 1b, S1), presumably indicating amacrine cell function during the rod response.

Homozygosity mapping using genome-wide genotypes from 11 family members indicated a $15.12-\mathrm{Mb}$ region of homozygosity on chromosome 1q23.1-q24.3 (Fig. S2a). Linkage analysis yielded a maximum two-point LOD score of $3.1(\theta=0)$ at rs6700867 (chr1:162.31 Mb; NCBI Build hg19). A statistically significant maximum multipoint LOD score of 3.6 was obtained at several markers from rs1538971 (chr1:161.68 Mb) to rs17350579 (chr1:172.07 Mb). Both the minimum critical region identified by linkage analysis and the homozygous region were flanked by markers rs2758684 (chr1:157.54 Mb) and rs859652 (chr1:172.65 Mb) and include $15.12 \mathrm{Mb}$ of sequence (Fig. S2b).

A DNA sample from the proband III-1 (Fig. 1a) underwent exome sequencing. Within the homozygous region on chromosome 1q23.1-q24.3, from the exome sequence data there are 14 homozygous variants with zero MAF in the ExAC database. Of the 14 rare variants, 13 variants were intergenic or intronic and occurred in dbSNP, and are unlikely to be disease-causal. On the other hand, a duplication variant c.355_356dupG (p.Glu119Glyfs*8) within ATF6 (NM_007348.3) was absent in both dbSNP and $\sim 120,000$ ExAC alleles. Additionally, the variant was not identified in 470 ethnically matched control chromosomes or in exome sequence data from 130 unrelated Pakistani individuals with non-ophthalmologic traits. Sanger sequencing of DNA samples from the remaining members of family MA28 confirmed co-segregation of the c.355_356dupG variant with ACHM (Fig. 1a, c). Two-point linkage analysis using genotypes for the c.355_356dupG variant yielded a statistically significant LOD score of 3.6 $(\theta=0)$.

The duplication of guanosine within exon 5 of ATF6 is predicted to alter the reading frame (Fig. S2c), thus resulting in the substitution of glutamic acid at position 119 with glycine followed by a frameshift. The variant introduces a premature nonsense codon before the amino acids encoding the basic region leucine zipper (BRLZ) and transmembrane (TM) domains (Fig. S3). Multiple sequence alignment of human ATF6 and related sequences from 96 species indicated that several amino acid residues within the BRLZ domain are highly conserved or identical across species (Fig. S3a). These residues are also identical or highly conserved in various human transcription factors, supporting the important role of the BRLZ domain in target gene activation (Fig. S3b). Molecular modeling at $\geq 90 \%$ confidence revealed that wild-type ATF6 is identical in sequence with other transcription factors by $45 \%$ at maximum, but the sequence identity is limited to the BRLZ domain (Fig. S3c-d). Comparison of wild-type and mutant models for ATF6 predicts removal of ordered structure particularly of the BRLZ and TM domains (Fig. S3d).

There are three alternatively spliced isoforms of human ATF6 (Fig. 1d, S2c). Isoform AK290498 has 16 exons and encodes a 670 -amino acid protein. Exon 16 is alternatively spliced in isoform AF005887 at the $3^{\prime}$ UTR but has the same coding region as in isoform A. Isoform AB208929 has 14 coding exons and encodes a 577-amino acid protein (Table S1). RT-PCR performed on commercially obtained RNA samples showed expression of all three ATF6 isoforms in human eye, particularly in retinal pigment epithelium cells (Fig. 1d). All three ATF6 isoforms include exons $1-5$ in sequence, thus the duplication variant is predicted to affect all three isoforms in the same manner (Fig. S2c).

The occurrence of multiple isoforms and ATF6 multimers in the eye is further confirmed by western blot analysis using anti-ATF6 antibodies on protein extract from adult C57BL/6J mouse retina (Fig. S3e). The anti-ATF6 antibodies were validated in heterologous cells. Immunofluorescent staining with anti-ATF6 antibodies overlapped with the signal produced by tagged ATF6 expressed in COS-7 cells (Fig. S4), which confirm the specificity of the antibody for ATF6. To explore the precise cellular localization in the eye, CD1 mouse retinae were examined for immunoreactivity with anti-ATF6 antibodies (Fig. 2, S5). ATF6 immunoreactivity is most prominent in the retinal ganglion cells. Staining of ATF6 was also observed in the retinal pigment epithelium, outer and inner segments of photoreceptor cells, inner and outer plexiform layers, as well as in the inner nuclear layer of neuronal retina (Fig. 2, S5).

ATF6 encodes a transcription factor that is initially synthesized as an ER-embedded transmembrane protein. To determine the effect of the ATF6 p.Glu119Glyfs*8 variant 
Fig. 2 Localization of ATF6 in wild-type mouse retina. Immunostaining in CD1 mouse retina with antibodies for DAPI (blue), rhodamine phalloidin (red) for actin-based structures and ATF6 (green). ATF6 strongly localized to the retinal ganglion cells (RGC), and had moderate staining of the outer and inner segments of photoreceptor cells (PR) and the inner plexiform (IPL), inner nuclear (INL) and outer plexiform (OPL) layers. ATF6 is weakly localized to the outer nuclear layer (ONL) which contains photoreceptor cell bodies (color figure online)

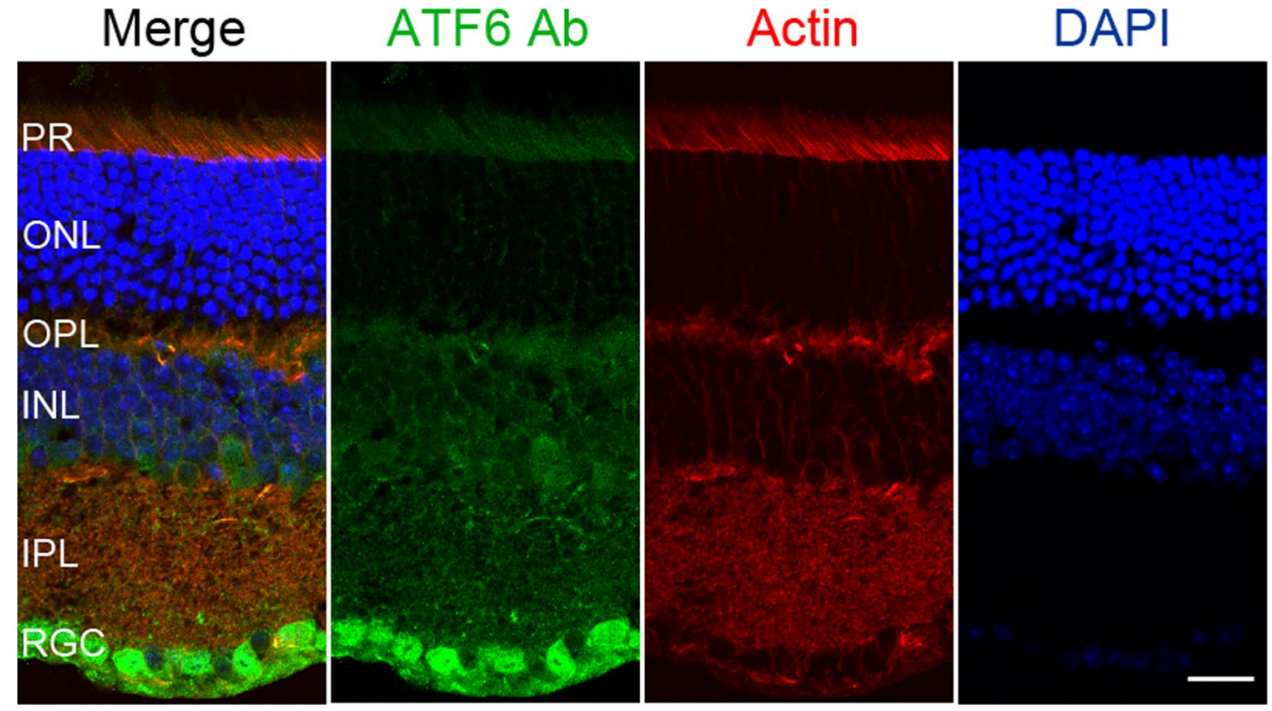

(a)

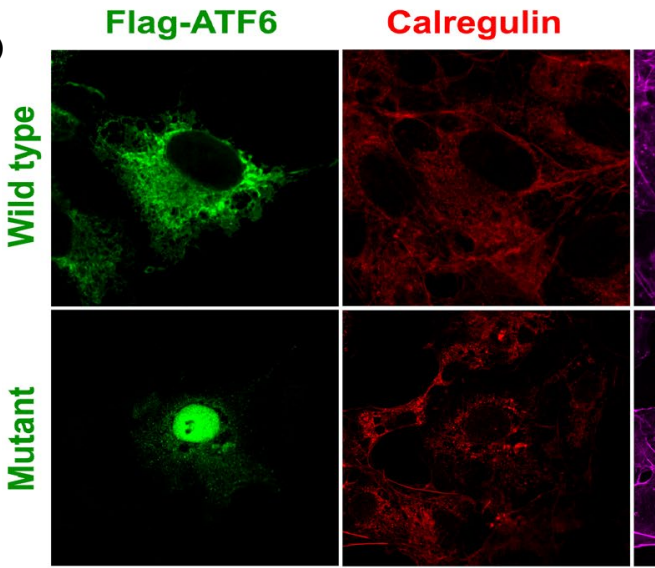

Actin

Merge

type and mutant ATF6 in transfected COS-7 cells. a

Flag-tagged wild-type ATF6 protein is expressed throughout the cytoplasm and localized in the endoplasmic reticulum. In contrast, the p.Glu119Glyfs*8 variant affected the stability, targeting and localization of ATF6, with expression confined to the nucleus and significantly reduced cytoplasmic localization. Scale bars $10 \mu \mathrm{m}$. b COS-7 cells were transiently transfected with the same quantity of wild-type or mutant ATF6 constructs. Protein extracts from the cell lysates were analyzed by western blot using an anti-ATF6 antibody. Flag-tagged wild-type ATF6 protein is demonstrated at the expected size $\sim 64 \mathrm{kDa}$ and with dimer formation. In contrast, mutant ATF6 had reduced protein size. GAPDH antibody was used as loading control. Quantification of the steady-state expression did not reveal any significant difference between the wild-type and mutant ATF6

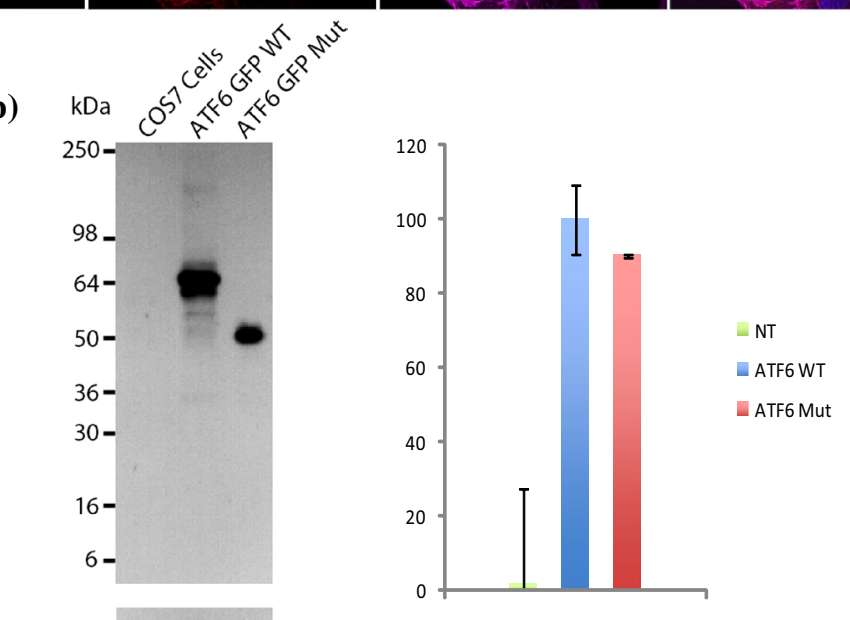

GAPDH on steady state levels and localization, COS-7 cells were co-transfected with GFP-tagged human ATF6 constructs. As expected, confocal imaging of the tagged wild-type ATF6 revealed expression throughout the cytoplasm and localization to ER (Fig. 3a). In contrast, mutant ATF6 with the p.Glu119Glyfs*8 variant had significantly reduced localization in the cytoplasm and was mainly confined to the nucleus (Fig. 3a). Western blot analysis and 
quantification, after normalization against the GAPDH expression level, revealed no significant difference in the steady state level of the p.Glu119Glyfs*8 mutant protein compared with wild-type ATF6 protein (Fig. 3b). Thus, in vitro, the p.Glu119Glyfs*8 variant affects the targeting and localization of ATF6.

\section{Discussion}

In this study, we identified a rare variant in ATF6 that segregates with the ACHM phenotype. ATF6 lies within an autosomal recessive cone-rod dystrophy (CORD8) locus that was previously mapped in another Pakistani family to an 11.53-cM region on chromosome 1q12-q24 (Khaliq et al. 2000; Ismail et al. 2006). The affected individuals of the CORD8 family also presented with color vision loss, severe photophobia and progressive deterioration of vision since childhood. While the affected members of the CORD8 family had night blindness (Khaliq et al. 2000), the affected individuals from our ACHM family had normal night vision. Mutations in four previously identified ACHM genes namely CNGA3, CNGB3, PDE6C and PDE6H can cause both ACHM and CORD (Huang et al. 2013; Li et al. 2014b). However, negative results from Sanger sequencing of all exons of ATF6 using DNA samples of two affected individuals from the CORD8 family ruled out the involvement of ATF6 in the etiology of CORD. It is possible that another gene within chromosome 1q is responsible for CORD. On the other hand, genome-wide mapping and exome sequencing provided strong evidence that the ATF6 c.355_356dupG (p.Glu119Glyfs*8) variant causes ACHM in family MA28.

Although ATF6 is ubiquitously expressed, atf $6^{-/-}$ zebrafish and both $A t f 6 \alpha^{-1-}$ and Atf $6 \beta^{-/-}$mice do not have obvious developmental abnormalities, while double knockout mice for Atf $6 \alpha^{-1-;} A t f 6 \beta^{-1-}$ were embryonic lethal (Wu et al. 2007; Yamamoto et al. 2007; Cinaroglu et al. 2011). There is also evidence that Atf $6 \alpha$-null mice subjected to high-fat diet develop glucose intolerance and after ischemic stroke have greater size of brain infarcts, both of which are explained by conditions of ER stress (Usui et al. 2012; Yoshikawa et al. 2015). In family MA28, there is no evidence of morphologic, cardiovascular, endocrine, or neurologic disease except for ACHM from early childhood. RT-PCR showed expression of all ATF6 isoforms in retina (Fig. 1d), which are predicted to be equally affected by the ATF6 c.355_356dupG variant. In humans, truncating ATF6 variants that affect all isoforms may result in specific phenotypes brought about by ER stress, which in the case of the retina may be due to light exposure or inherited deficiencies (Thapa et al. 2012).

In general, cells respond to ER stress by activating the unfolded protein response (UPR) to facilitate ER protein folding and reduce misfolded protein levels (Chiang et al. 2012). In retinas exposed to ER stress due to excessive light injury, ATF6 mediates early UPR which serves to suppress ER stress and restore homeostasis (Nakanishi et al. 2013). ATF6 also activates IRE1 which mediates late UPR, during which ATF6 activation is decreased and severe or prolonged stress can result in apoptotic cell death (Lin et al. 2007; Nakanishi et al. 2013). ER stress-associated apoptotic cell death has been implicated as a common pathway for retinal degenerative disease, including ACHM due to gene defects in photoreceptor cyclic nucleotide-gated channels CNGA3 and CNGB3 (Thapa et al. 2012). Rod-cone photoreceptor dysfunction has been clearly demonstrated in the presence of mutation in other ACHM genes (Kohl et al. 2000; Thiadens et al. 2010; Aboshiha et al. 2014). Expression of previously identified ACHM genes has been equally distributed throughout the retina. Likewise, ATF6 has ubiquitous retinal expression with strongest localization in retinal ganglion cells and moderate staining at the retinal pigment epithelium, photoreceptor outer and inner segments, outer and inner plexiform and inner nuclear layers (Fig. 2, S5).

While all these cell layers that express ATF6 are widely known to be involved in color vision, absence of photopic a-waves in the ffERG of the ACHM proband from family MA28 supports functional loss of cone photoreceptors due to the ATF6 duplication variant (Fig. 1b). The role of the ATF6 pathway in retinal ER stress response was previously described within photoreceptor cells (Yang et al. 2007, 2008; Saito 2014). In photoreceptors, ATF6 mRNA and protein expression is upregulated after light or drug exposure (Chiang et al. 2012; Li et al. 2014a). On the other hand, photopic b-wave diminution in the ffERG can be due to the absence of cone photoreceptor response and/or Müller and on-bipolar cell dysfunction at the inner retinal layers. Several studies have demonstrated the ER stress response through ATF6 within the inner retina. In particular, ER stress within normal and disease contexts (e.g., drug exposure, genetic loss of ER transmembrane protein, diabetic retinopathy) resulted in increased ATF6 expression in Müller glial and retinal ganglion cells (Yoshikawa et al. 2011; Wu et al. 2012; Miyagi et al. 2013; Ha et al. 2014; Zode et al. 2014). Although ffERG does not capture ganglion cell function, it is interesting to note that retinal ganglion cells primarily synapse with bipolar cells within the cone system. Retinal ganglion cells have been identified as the retinal cells most affected by ER stress due to diabetes (Yang et al. 2013) and glaucoma (Zode et al. 2014), and color vision defects have been documented in both acquired disease states (Lin and Yang 2010; Shoji et al. 2011). The unique retinal staining pattern of ATF6 with strongest localization to ganglion cells might imply that the ATF6 pathway within the retinal ganglion cells plays a major role in color vision. 
In response to ER stress, ATF6 undergoes proteolysis to release its BRLZ domain, which binds to the ER stress response element to activate expression of downstream target genes within the nucleus (Haze et al. 1999). The interplay between transcription factors and target genes involved in the ER stress pathway is thought to allow fine-tuning of the UPR response that is cell or tissue specific (Saito 2014). Although MutationTaster (Schwarz et al. 2014) predicts the ATF6 frameshift variant to initiate nonsense-mediated decay, immunolocalization studies in COS-7 cells overexpressing the mutant ATF6 cDNA construct revealed the production of a smaller protein that is predominantly mislocalized to the nucleus (Fig. 3). Reduced cytoplasmic expression of truncated protein is to be expected given the loss of the TM domain that anchors ATF6 to the ER (Haze et al. 1999). While ATF6 is required to translocate to the nucleus to activate ER stress genes, the lack of a functional BRLZ domain in the truncated ATF6 protein should preclude DNA binding and UPR activation. Our experiments therefore predict that in vivo ATF6 protein with the frameshift variant is not reduced in overall amount of expression but is truncated, mislocalized and non-functional.

In conclusion, we identified a variant in ATF6 as the underlying cause of autosomal recessive achromatopsia. This finding lends further support to the importance of the ATF6 pathway in retinal function. ATF6 is localized throughout the retina, with strongest staining within retinal ganglion cells. Taken together, our findings imply that the ATF6 pathway is essential in color vision not just within photoreceptors but also within the inner retina, and is a potential target for treatment for either inherited or acquired retinal diseases.

Acknowledgments The authors thank the family members who participated in this study. This work was supported by the Higher Education Commission of Pakistan (Project \#420) and the National Institutes of Health Grants R01 DC012564, R56 AR065483 and U54 HG006493.

Open Access This article is distributed under the terms of the Creative Commons Attribution 4.0 International License (http://creativecommons.org/licenses/by/4.0/), which permits unrestricted use, distribution, and reproduction in any medium, provided you give appropriate credit to the original author(s) and the source, provide a link to the Creative Commons license, and indicate if changes were made.

\section{Appendix}

\section{University of Washington Center for Mendelian Genomics}

Michael J. Bamshad ${ }^{1,2}$, Jay Shendure ${ }^{1}$, and Deborah A. Nickerson ${ }^{1}$, Gonçalo R. Abecasis ${ }^{4}$, Peter Anderson ${ }^{1}$, Marcus Annable ${ }^{1}$, Mallory Beightol ${ }^{1}$, Brian L. Browning ${ }^{1}$, Kati J. Buckingham ${ }^{1}$, Christina Chen ${ }^{1}$, Jennifer Chin ${ }^{1}$, Jessica X. Chong ${ }^{1}$, Gregory M. Cooper ${ }^{5}$, Colleen Davis ${ }^{1}$, Lindsay
Felker ${ }^{1}$, Christopher Frazar ${ }^{1}$, David Hanna ${ }^{1}$, Zongxiao $\mathrm{He}^{3}$, Preti Jain ${ }^{5}$, Gail P. Jarvik ${ }^{1}$, Eric Johanson ${ }^{1}$, Goo Jun ${ }^{4}$, Martin Kircher ${ }^{1}$, Tom Kolar ${ }^{1}$, Suzanne M. Leal ${ }^{3}$, Daniel Luksic $^{1}$, Margaret J. McMillin ${ }^{1}$, Sean McGee ${ }^{1}$, Brenton Munson $^{1}$, Brian J. O'Roak ${ }^{1}$, Bryan Paeper ${ }^{1}$, Karynne Patterson ${ }^{1}$, Eric Phillips ${ }^{1}$, Jessica Pijoan ${ }^{1}$, Christa Poel ${ }^{1}$, Peggy D. Robertson $^{1}$, Regie Santos-Cortez ${ }^{3}$, Tristan Shaffer ${ }^{1}$, Cindy Shephard $^{1}$, Deborah L. Siegel ${ }^{1}$, Joshua D. Smith ${ }^{1}$, Jeffrey C. Staples ${ }^{1}$, Holly K. Tabor ${ }^{1,2}$, Monica Tackett ${ }^{1}$, Gao Wang ${ }^{3}$, and Qian $\mathrm{Yi}^{1}$

${ }^{1}$ University of Washington

${ }^{2}$ Seattle Children's Hospital

${ }^{3}$ Baylor College of Medicine

${ }^{4}$ University of Michigan

${ }^{5}$ HudsonAlpha Institute of Technology

\section{References}

Abecasis GR, Cherny SS, Cookson WO, Cardon LR (2002) Merlinrapid analysis of dense genetic maps using sparse gene flow trees. Nat Genet 30:97-101

Aboshiha J, Dubis AM, Cowing J, Fahy RT, Sundaram V, Bainbridge JW, Ali RR, Dubra A, Nardini M, Webster AR, Moore AT, Rubin G, Carroll J, Michaelides M (2014) A prospective longitudinal study of retinal structure and function in achromatopsia. Investig Ophthalmol Vis Sci 55:5733-5743

Chang B, Grau T, Dangel S, Hurd R, Jurklies B, Sener EC, Andreasson S, Dollfus H, Baumann B, Bolz S, Artemyev N, Kohl S, Heckenlively J, Wissinger B (2009) A homologous genetic basis of the murine cpfl1 mutant and human achromatopsia linked to mutations in the PDE6C gene. Proc Natl Acad Sci USA 106:19581-19586

Chiang WC, Hiramatsu N, Messah C, Kroeger H, Lin JH (2012) Selective activation of ATF6 and PERK endoplasmic reticulum stress signaling pathways prevent mutant rhodopsin accumulation. Investig Ophthalmol Vis Sci 53:7159-7166

Cinaroglu A, Gao C, Imrie D, Sadler KC (2011) Activating transcription factor 6 plays protective and pathological roles in steatosis due to endoplasmic reticulum stress in zebrafish. Hepatology 54:495-508

Ha Y, Shanmugam AK, Markand S, Zorrilla E, Ganapathy V, Smith SB (2014) Sigma receptor 1 modulates ER stress and Bcl2 in murine retina. Cell Tissue Res 356:15-27

Haze K, Yoshida H, Yanagi H, Yura T, Mori K (1999) Mammalian transcription factor ATF6 is synthesized as a transmembrane protein and activated by proteolysis in response to endoplasmic reticulum stress. Mol Biol Cell 10:3787-3799

Huang L, Zhang Q, Li S, Guan L, Xiao X, Zhang J, Jia X, Sun W, Zhu Z, Gao Y, Yin Y, Wang P, Guo X, Wang J, Zhang Q (2013) Exome sequencing of 47 Chinese families with cone-rod dystrophy: mutations in 25 known causative genes. PLoS One 8:e65546

Ismail M, Abid A, Anwar K, Mehdi SQ, Khaliq S (2006) Refinement of the locus for autosomal recessive cone-rod dystrophy (CORD8) linked to chromosome 1q23-q24 in a Pakistani family and exclusion of candidate genes. J Hum Genet $51: 827-831$

Kelley LA, Sternberg MJ (2009) Protein structure prediction on the Web: a case study using the Phyre server. Nat Protoc 4:363-371 
Khaliq S, Hameed A, Ismail M, Anwar K, Leroy BP, Mehdi SQ, Payne AM, Bhattacharya SS (2000) Novel locus for autosomal recessive cone-rod dystrophy CORD8 mapping to chromosome 1q12-q24. Investig Ophthalmol Vis Sci 41:3709-3712

Kohl S, Coppieters F, Meire F, Schaich S, Roosing S, Brennenstuhl C, Bolz S, van Genderen MM, Riemslag FC, European Retinal Disease Consortium, Lukowski R, den Hollander AI, Cremers FP, De Baere E, Hoyng CB, Wissinger B (2012) A nonsense mutation in PDE6H causes autosomal-recessive incomplete achromatopsia. Am J Hum Genet 91:527-532

Kohl S, Marx T, Giddings I, Jägle H, Jacobson SG, Apfelstedt-Sylla E, Zrenner E, Sharpe LT, Wissinger B (1998) Total colour blindness is caused by mutations in the gene encoding the alpha-subunit of the cone photoreceptor cGMP-gated cation channel. Nat Genet 19:257-259

Kohl S, Baumann B, Broghammer M, Jägle H, Sieving P, Kellner U, Spegal R, Anastasi M, Zrenner E, Sharpe LT, Wissinger B (2000) Mutations in the CNGB3 gene encoding the beta-subunit of the cone photoreceptor cGMP-gated channel are responsible for achromatopsia (ACHM3) linked to chromosome 8q21. Hum Mol Genet 9:2107-2116

Kohl S, Baumann B, Rosenberg T, Kellner U, Lorenz B, Vadalà M, Jacobson SG, Wissinger B (2002) Mutations in the cone photoreceptor G-protein alpha-subunit gene GNAT2 in patients with achromatopsia. Am J Hum Genet 71:422-425

Li H, Durbin R (2009) Fast and accurate short read alignment with Burrows-Wheeler transform. Bioinformatics 25:1754-1760

Li GY, Fan B, Jiao YY (2014a) Rapamycin attenuates visible lightinduced injury in retinal photoreceptor cells via inhibiting endoplasmic reticulum stress. Brain Res 1563:1-12

Li S, Huang L, Xiao X, Jia X, Guo X, Zhang Q (2014b) Identification of CNGA3 mutations in 46 Families: common cause of achromatopsia and cone-rod dystrophies in Chinese patients. JAMA Ophthalmol 132:1076-1083

Lin JC, Yang MC (2010) Correlation of visual function with healthrelated quality of life in glaucoma patients. J Eval Clin Pract 16:134-140

Lin JH, Li H, Yasumura D, Cohen HR, Zhang C, Panning B, Shokat KM, Lavail MM, Walter P (2007) IRE1 signaling affects cell fate during the unfolded protein response. Science 318:944-949

Matise TC, Chen F, Chen W, De La Vega FM, Hansen M, He C, Hyland FC, Kennedy GC, Kong X, Murray SS, Ziegle JS, Stewart WC, Buyske S (2007) A second-generation combined linkage physical map of the human genome. Genome Res 17:1783-1786

McKenna A, Hanna M, Banks E, Sivachenko A, Cibulskis K, Kernytsky A, Garimella K, Altshuler D, Gabriel S, Daly M, DePristo MA (2010) The genome analysis toolkit: a MapReduce framework for analyzing next-generation DNA sequencing data. Genome Res 20:1297-1303

Michaelides M, Hunt DM, Moore AT (2004) The cone dysfunction syndromes. Br J Ophthalmol 88:291-297

Miyagi H, Kanemoto S, Saito A, Asada R, Iwamoto H, Izumi S, Kido M, Gomi F, Nishida K, Kiuchi Y, Imaizumi K (2013) Transcriptional regulation of VEGFA by the endoplasmic reticulum stress transducer OASIS in ARPE-19 cells. PLoS One 8:e55155

Nakanishi T, Shimazawa M, Sugitani S, Kudo T, Imai S, Inokuchi Y, Tsuruma K, Hara H (2013) Role of endoplasmic reticulum stress in light-induced photoreceptor degeneration in mice. J Neurochem 125:111-124

O'Connell JR, Weeks DE (1998) PedCheck: a program for identification of genotype incompatibilities in linkage analysis. Am J Hum Genet 63:259-266

Saito A (2014) Physiological functions of endoplasmic reticulum stress transducer OASIS in central nervous system. Anat Sci Int 89:11-20
Saqib MA, Awan BM, Sarfraz M, Khan MN, Rashid S, Ansar M (2011) Genetic analysis of four Pakistani families with achromatopsia and a novel S4 motif mutation of CNGA3. Jpn J Ophthalmol 55:676-680

Schwarz JM, Cooper DN, Schuelke M, Seelow D (2014) MutationTaster2: mutation prediction for the deep-sequencing age. Nat Methods 11:361-362

Seelow D, Schuelke M, Hildebrandt F, Nurnberg P (2009) HomozygosityMapper-an interactive approach to homozygosity mapping. Nucleic Acids Res 37:593-599

Shoji T, Sakurai Y, Sato H, Chihara E, Takeuchi M (2011) Do type 2 diabetes patients without diabetic retinopathy or subjects with impaired fasting glucose have impaired colour vision? The Okubo Color Study Report. Diabet Med 28:865-871

Sievers F, Wilm A, Dineen D, Gibson TJ, Karplus K, Li W, Lopez R, McWilliam H, Remmert M, Söding J, Thompson JD, Higgins DG (2011) Fast, scalable generation of high-quality multiple sequence alignments using clustal Omega. Mol Syst Biol 7:539

Silberstein M, Tzemach A, Dovgolevsky N, Fishelson M, Schuster A, Geiger D (2006) Online system for faster multipoint linkage analysis via parallel execution on thousands of personal computers. Am J Hum Genet 78:922-935

Thapa A, Morris L, Xu J, Ma H, Michalakis S, Biel M, Ding XQ (2012) Endoplasmic reticulum stress-associated cone photoreceptor degeneration in cyclic nucleotide-gated channel deficiency. J Biol Chem 287:18018-18029

Thiadens AA, Somervuo V, van den Born LI, Roosing S, van Schooneveld MJ, Kuijpers RW, van Moll-Ramirez N, Cremers FP, Hoyng CB, Klaver CC (2010) Progressive loss of cones in achromatopsia: an imaging study using spectral-domain optical coherence tomography. Investig Ophthalmol Vis Sci 51:5952-5957

Usui M, Yamaguchi S, Tanji Y, Tominaga R, Ishigaki Y, Fukumoto M, Katagiri H, Mori K, Oka Y, Ishihara H (2012) Atf6 $\alpha$-null mice are glucose intolerant due to pancreatic $\beta$-cell failure on a highfat diet but partially resistant to diet-induced insulin resistance. Metabolism 61:1118-1128

Wu J, Rutkowski DT, Dubois M, Swathirajan J, Saunders T, Wang J, Song B, Yau GDY, Kaufman RJ (2007) ATF6 $\alpha$ optimizes long-term endoplasmic reticulum function to protect cells from chronic stress. Dev Cell 13:351-364

Wu J, Sun P, Zhang X, Liu H, Jiang H, Zhu W, Wang H (2012) Inhibition of GPR40 protects MIN6 $\beta$ cells from palmitate-induced ER stress and apoptosis. J Cell Biochem 113:1152-1158

Yamamoto K, Sato T, Matsui T, Sato M, Okada T, Yoshida H, Harada A, Mori K (2007) Transcriptional induction of mammalian ER quality control proteins is mediated by single or combined action of ATF $6 \alpha$ and XBP1. Dev Cell 13:165-176

Yang LP, Wu LM, Guo XJ, Tso MO (2007) Activation of endoplasmic reticulum stress in degenerating photoreceptors of the $\mathrm{rd} 1$ mouse. Investig Ophthalmol Vis Sci 48:5191-5198

Yang LP, Wu LM, Guo XJ, Li Y, Tso MO (2008) Endoplasmic reticulum stress is activated in light-induced retinal degeneration. J Neurosci Res 86:910-919

Yang C, Diiorio P, Jurczyk A, O’Sullivan-Murphy B, Urano F, Bortell R (2013) Pathological endoplasmic reticulum stress mediated by the IRE1 pathway contributes to pre-insulitic beta cell apoptosis in a virus-induced rat model of type 1 diabetes. Diabetologia 56:2638-2646

Yoshikawa T, Ogata N, Izuta H, Shimazawa M, Hara H, Takahashi K (2011) Increased expression of tight junctions in ARPE-19 cells under endoplasmic reticulum stress. Curr Eye Res 36:1153-1163

Yoshikawa A, Kamide T, Hashida K, Ta HM, Inahata Y, TakaradaIemata M, Hattori T, Mori K, Takahashi R, Matsuyama T, Hayashi Y, Kitao Y, Hori O (2015) Deletion of Atf6 $\alpha$ impairs astroglial activation and enhances neuronal death following brain ischemia in mice. J Neurochem 132:342-353 
Zode GS, Sharma AB, Lin X, Searby CC, Bugge K, Kim GH, Clark AF, Sheffield VC (2014) Ocular-specific ER stress reduction rescues glaucoma in murine glucocorticoid-induced glaucoma. J Clin Investig 124:1956-1965

\section{Web resources}

Burrows-Wheeler Aligner, http://bio-bwa.sourceforge.net/ Clustal Omega, http://www.ebi.ac.uk/Tools/msa/clustalo/ dbSNP, http://www.ncbi.nlm.nih.gov/SNP/

Exome Aggregation Consortium (ExAC), http://exac.broadinstitute. org/

Genome Analysis Toolkit (GATK), https://www.broadinstitute.org/ gatk/
HomozygosityMapper, http://www.homozygositymapper.org/ MERLIN, http://www.sph.umich.edu/csg/abecasis/Merlin/

MutationTaster, http://www.mutationtaster.org/

Phyre2, http://www.sbg.bio.ic.ac.uk/phyre2/html/page.cgi?id=index Primer3, http://frodo.wi.mit.edu/

RCSB Protein Data Bank (PDB), http://www.rcsb.org/pdb/home/home. do

SeattleSeq Annotation 137, http://snp.gs.washington.edu/SeattleSeq Annotation137/

Superlink Online SNP 1.1, http://cbl-hapw.cs.technion.ac.il/superlinksnp/

UCSC Genome Bioinformatics, http://genome.ucsc.edu/

UniProt BLAST, http://www.uniprot.org/blast/ 